\title{
Organization of microbeads in Leidenfrost drops ${ }^{\dagger}$
}

\author{
Laurent Maquet, ${ }^{* a}$ Pierre Colinet, ${ }^{b}$ and Stéphane Dorbolo ${ }^{a}$
}

\author{
Received Xth XXXXXXXXXX 20XX, Accepted Xth XXXXXXXXX 20XX \\ First published on the web Xth $X X X X X X X X X X 200 X$ \\ DOI: 10.1039/b000000x
}

We investigated the organization of micrometric hydrophilic beads (glass or basalt) immersed in Leidenfrost drops. Starting from a large volume of water compared to the volume of the beads, while the liquid evaporates, we observed that the grains are eventually trapped at the interface of the droplet and accumulate. At a moment, the grains entirely cover the droplet. We measured the surface area at this moment as a function of the total mass of particles inserted in the droplet. We concluded that the grains form a monolayer around the droplet assuming (i) that the packing of the beads at the surface is a random close packing and (ii) that the initial surface of the drop is larger than the maximum surface that the beads can cover. Regarding the evaporation dynamics, the beads are found to reduce the evaporation rate of the drop. The slowdown of the evaporation is interpreted as being the consequence of the dewetting of the particles located at the droplet interface which make of the effective surface of evaporation smaller. As a matter of fact, contact angles of the beads with the water deduced from the evaporation rates are consistent with contact angles of beads directly measured at the air - water interface in a container.

\section{Introduction}

When a millimetric drop of liquid is released on a plate whose temperature is just above the boiling temperature of the liquid, it is well known that the drop evaporates in a short time, around typically one second. However, if the plate temperature is far above this temperature, one can observe a drop that takes several minutes to evaporate. The drop is not anymore in contact with the plate but levitates over a thin film of its own vapor which thermally isolates the drop. Since this discovery, more than two centuries ago ${ }^{1}$, this effect, known as the Leidenfrost effect, keeps focusing investigations nowadays $^{2}$. Indeed, after a decade during which the phenomenon was revisited, the Leidenfrost effect is now used to self-propel droplets over ratchets ${ }^{3-5}$ or to carry small objects (centimetric) thanks to the quasi-frictionless transport ${ }^{6}$. But there is still much to do to understand fundamental aspects of these drops and especially their interactions with particles.

As a matter of fact, many studies on the Leidenfrost effect concern the case of pure liquids (e.g. studies on the Leidenfrost Point ${ }^{7-9}$, on the shape of the drops ${ }^{10,11}$, on their oscillations ${ }^{12}$ ). Yet, it has been shown that colloidal suspensions of carboxylate-modified polystyrene beads present some interesting buckling properties when they are dried in a Leidenfrost

† Electronic Supplementary Information (ESI) available: movie of a Leidenfrost drop with basalt beads organizing at its surface. See DOI: 10.1039/b000000x/

${ }^{a}$ GRASP, Département de Physique B5, Université de Liège, B-4000 Liège, Belgium.Tel: +3243663707; E-mail:lmaquet@doct.ulg.ac.be

${ }^{b}$ TIPs, Université Libre de Bruxelles, CP165/67, avenue F.D. Roosevelt 50, B-1050 Bruxelles, Belgium. state $^{13}$. Due to the low diffusion rate of the particles, the volume fraction of beads increases near the interface and a shell is formed. Besides, nanoparticles can be self-organized by Leidenfrost droplets moving or impacting over substrates ${ }^{14}$. Finally, it has been shown that drops coated by hydrophobic microparticles, i.e. liquid marbles ${ }^{15,16}$, can also levitate ${ }^{17}$. Moreover, the critical temperature above which the Leidenfrost effect takes place does not exist for liquid marbles, i.e. the evaporation duration of a liquid marble continuously decreases with the temperature. The hydrophobic particles actually form a porous layer that isolates the liquid phase from the plate $^{17}$.

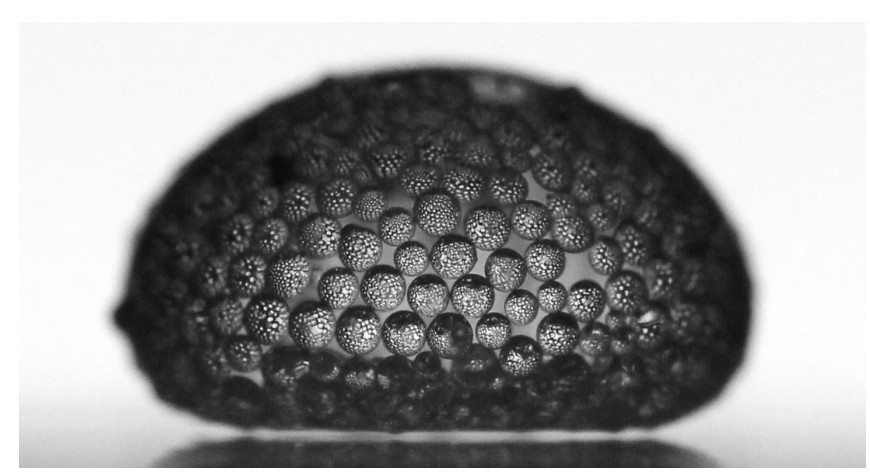

Fig. 1 Picture of a centimetric Leidenfrost drop covered by glass beads of mean radius $R_{b}=150 \mu \mathrm{m}$. The dots in the beads are due to the refraction of the beads on the hidden face of the drop.

In contrast with the case studied by Aberle et al. ${ }^{17}$, we focused here on hydrophilic particles when they are introduced 
into Leidenfrost drops. Note that the definition of the term hydrophilic will be clarified in the present framework. We will show that during the evaporation of the Leidenfrost droplet, the grains move towards the interface of the droplet where they remain trapped because of a partial dewetting of the grain surface. Eventually, the grains entirely cover the droplet forming an object that is analogous to a liquid marble (see Fig. 1). In the following section, we describe the experimental conditions for which we obtained this self-organized layer of grains. The experimental results consist in the measurements of the fraction of the grains that are trapped by the interface and the influence of the beads on the evaporation rate of the droplet. To interpret the time evolution of the droplet volume, a model based on the scalings proposed by Biance et al. ${ }^{18}$ has been developed considering that the global density of the drop increases with time and that the presence of grains at the interface reduces the effective surface for evaporation.

\section{Experimental details}

A polished aluminum plate was placed on a heating surface. A PID controller was used to keep the temperature of the plate at the set point within a degree. The plate was $1 \mathrm{~cm}$ thick at the edges and was very slightly curved (radius of curvature $\sim 5 \mathrm{~m}$ ). This curvature prevents the Leidenfrost drops from moving away.

Different kinds of beads were used in these experiments. The characteristics of these beads are specified in Table 1. The glass beads were commercial SiLibeads grains type $\mathrm{S}$ (soda lime). Their density $\rho_{b}$ is $2500 \mathrm{~kg} / \mathrm{m}^{3}$. Three size classes were used. The first class were glass beads with radius $R_{b}$ ranging between $20 \mu \mathrm{m}$ and $35 \mu \mathrm{m}$, the second between $45 \mu \mathrm{m}$ and $75 \mu \mathrm{m}$, and the third between $100 \mu \mathrm{m}$ and $200 \mu \mathrm{m}$. The basalt grains were Whitehouse Scientific basalt beads. Their density $\rho_{b}$ is $2900 \mathrm{~kg} / \mathrm{m}^{3}$. Ninety percent of the radius beads ranging between $53 \mu \mathrm{m}$ and $62.5 \mu \mathrm{m}$. In the following, the beads will be called by their associated number in Table 1 .

\begin{tabular}{|c|c|c|c|c|}
\hline & Matter & Density $\left(\mathrm{kg} / \mathrm{m}^{3}\right)$ & Radii range $(\mu \mathrm{m})$ & $\Theta_{d}$ \\
\hline \hline I & Glass & 2500 & $20-35$ & - \\
\hline II & Glass & 2500 & $45-75$ & $26^{\circ}$ \\
\hline III & Glass & 2500 & $100-200$ & $31^{\circ}$ \\
\hline IV & Basalt & 2900 & $53-62.5$ & $30^{\circ}$ \\
\hline
\end{tabular}

Table 1 The different types of beads used throughout the experiments.

In this table, we also report the contact angles of the beads with water. These angles are measured by direct imaging. To measure them, some beads are poured in a container filled with water at $85^{\circ} \mathrm{C}$ and we look at the beads with binoculars at
X5 directed paralel to the interface, just under it. Note that the measurement of the contact angle of the smallest beads was not possible with this method. The results for glass beads are consistent with common values of the angle of contact of water on glass 19,20 .

Both glass and basalt beads can be qualified as hydrophilic as their contact angle is below $90^{\circ}$. They can also be qualified as hydrophilic in the sense that when a water droplet is released over a bed of these particles, the droplet is observed to impregnate within a second in the bed of beads ${ }^{21}$. On the other hand, when dry beads are gently put at the surface of a water pool, the particles float, i.e. the contact line of the liquid remains pinned on the surface of the beads leaving a part of the beads dry. The flotability is due to the combination of capillary forces and buoyancy. Indeed, when the wettability of the beads is increased, e.g. using ethanol instead of water, our beads quickly sink when gently poured over an interface. In Leidenfrost drops made of ethanol, the beads stay mixed for the whole evaporation time. Furthermore, the differential evaporation of the ethanol and water in mixed drops makes impossible the methodology applied by Whitby et al. for sessile drops on bed of particles ${ }^{21}$. It is important to note that the following observations concern only grains that can float at the surface of the liquid.

The experimental procedure was the following. Drops of bidistilled water were made using a syringe. Their initial volume was $1 \mathrm{ml}$. The beads were then poured from the top through a glass funnel into the drop quickly after its release on the hot plate. The grains were previously weighted on a balance with a precision of $0.1 \mathrm{mg}$. As a few beads can sometimes fall around the drop, the uncertainty on the mass of beads inserted into the drops, $M$, is estimated to $0.2 \mathrm{mg}$.

We used a camera with a high resolution (5184 x 3456), i.e. Canon 600D. The captured images enabled extracting the geometrical properties of the drops (radii, surfaces, volumes) from sideviews with less than $10 \%$ relative uncertainties. A typical image is shown in Fig. 4(a).

\section{Results and discussion}

The initial volume of the droplet plays an important role. This volume must be much larger than the total volume of the grains in order to let the grain self-organize. Quickly after the insertion of the beads in a Leidenfrost drop, they are trapped at the interface of the drop. In large drops, they cover firstly a stripe at the basis of the drop likely where the vapor film is the thinest. In small drops, they directly cover the bottom face of the drop. Then, the evaporation of the drop occurs and the volume decreases. The beads remain trapped and cover the drop more and more, as its surface decreases, until the beads completely cover it. A partial coating is shown in Fig. 1. Looking closely at the interface, a roughness can be seen indicating that 
the particles emerges, i.e. are partially dried. As they do on the surface of water in a tank, and even though they experience a total wetting during their insertion, they float.

After the drop is completely covered, the surface available is not sufficient for the trapped particles. First the surface buckles before the grains start to sink in the bulk of the drop because of the frustration. Finally, the drop contacts the substrate. The droplet ends its contraction and looks like a sphere flattened by the gravity ${ }^{\dagger}$ as a liquid marble would $\mathrm{do}^{17}$.

\subsection{Liquid droplet wrapped in a monolayer of grains}

Observing the drops, e.g. on Fig. 1, the beads seem to form a monolayer at the surface, which is analogous to a liquid marble. It is important to contrast the obtention of these coated droplet with previous reported results concerning the spontaneous coating of drops by grains. Indeed, such a phenomenon has already been observed ${ }^{21,22}$, but in that case, the grains were hydrophobic in the sense that when a pure water droplet is deposited on a bed of these grains, the droplet does not penetrate the bed (see our discussion in Section 2). In the present case, the grains first sink to the bottom of the Leidenfrost droplet. They are first completely immersed and consequently, totally wetted. Then, due to evaporation, the grains partially dewet and are trapped by the interface.

To estimate the fraction of grains trapped at the interface, we need to compare the surface that the beads can cover $S_{\text {beads }}$ to the surface $S_{d r o p}$ available for the particles trapped at the surface at the moment when the drop is fully covered. $S_{\text {beads }}$ is given by

$$
S_{\text {beads }}=\frac{N_{b} \pi R_{b}^{2}}{\phi_{0}}
$$

where $N_{b}$ is the number of beads, $R_{b}$ their mean radius, and $\phi_{0}$ is the surface fraction of the packing. This latter was measured using high-contrast images with fluorescein and basalt beads (see Fig. 4(a)). The value found is $0.80 \pm 0.03$ which is close to the surface fraction of a random close packing of polydisperse hard disks ${ }^{23,24}$. The number $N_{b}$ of beads inserted in a drop is given by the ratio between the total mass of the beads inserted and the mean mass of a glass bead, namely

$$
N_{b}=\frac{3 M}{4 \pi R_{b}^{3} \rho_{b}}
$$

Thus, the inverse ratio of beads trapped at the interface, $\Gamma$, can be expressed as

$$
\Gamma=\frac{S_{\text {beads }}}{S_{\text {drop }}}=\frac{3 M}{4 R_{b} \rho_{b} \phi_{0}} \frac{1}{S_{\text {drop }}}
$$

We measured the surface of drops at the moment when the grains entirely cover the droplet for various quantity of beads and for the four kinds of beads described above. The calculated surface ratio $\Gamma$ is reported as a function of the mass of grains in Fig. 2 (see legend for the different grains considered). The present results are the average values over two experiments. The results show that $\Gamma$ remains clearly under 1.5 for all kind of beads used whatever the mass of inserted beads. Most of the grains always remain trapped at the interface. Equivalently, this means that at the moment where the drop becomes fully covered, less than $1 / 3$ of the beads are in the liquid bulk.

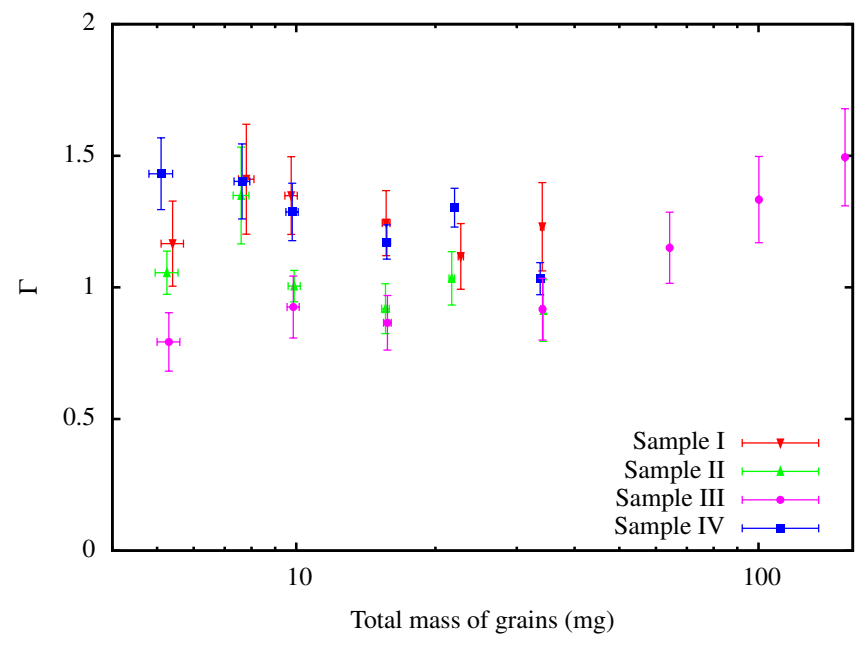

Fig. 2 The ratio between the surface that the beads can cover and the surface of the drop $\Gamma$ at the moment when it becomes fully covered as a function of the mass of grains inserted in the Leidenfrost droplet (initially $1 \mathrm{ml}$ ).

According to observations, two conditions are required to obtain a droplet covered by a monolayer of grains. First, the grains are able to float at the upper part of the interface. Second, the volume of the inserted grains must be much smaller than the initial volume of the Leidenfrost droplet. However, the initial size of the Leidenfrost droplet is limited. Indeed, for large drops, a Rayleigh-Taylor instability arises at the bottom of the drop ${ }^{18,25}$. For water, the instability occurs when the droplet radius exceeds $R_{c} \simeq 9.6 \mathrm{~mm}^{18}$. Above this value, the bottom of the droplet presents a pronounced annular neck in which the particles are trapped. Moreover, the bursts of the vapor bubble growing from the instability completely disturb the layer of beads and inhibits its formation. To avoid this, the initial volume of our drops corresponds to an initial radius $\left(R_{i} \simeq 7.5 \mathrm{~mm}\right)$ that is below the critical radius above which the instability occurs. 


\subsection{Influence of the monolayer on the evaporation rate.}

Given that the bottom surface of the drop is covered by the beads within the first seconds after their insertion, one wonders how the evaporation of these drops is affected. Indeed, similar objects, i.e. drops coated by hydrophobic particles, were observed to evaporate faster than a droplet with the same surface $^{26}$. To characterize the evaporation of our drops, we measured the variation of the volume of the drops as a function of time using image analysis. The substrate in this experiment is maintained at $300^{\circ} \mathrm{C}$. The results are presented in Fig. 3. We started with three drops of pure water of same initial volume and averaged their volume at each time (red squares). Then we ran the same experiment with drops loaded with $7.5 \pm 0.3 \mathrm{mg}$ of grains (green crosses) and with $30 \pm 0.3 \mathrm{mg}$ of grains (blue stars). The grains used in this experiment were from sample II. The evaporation of drops loaded with particles is observed to be systematically slower than the evaporation of pure water drops.

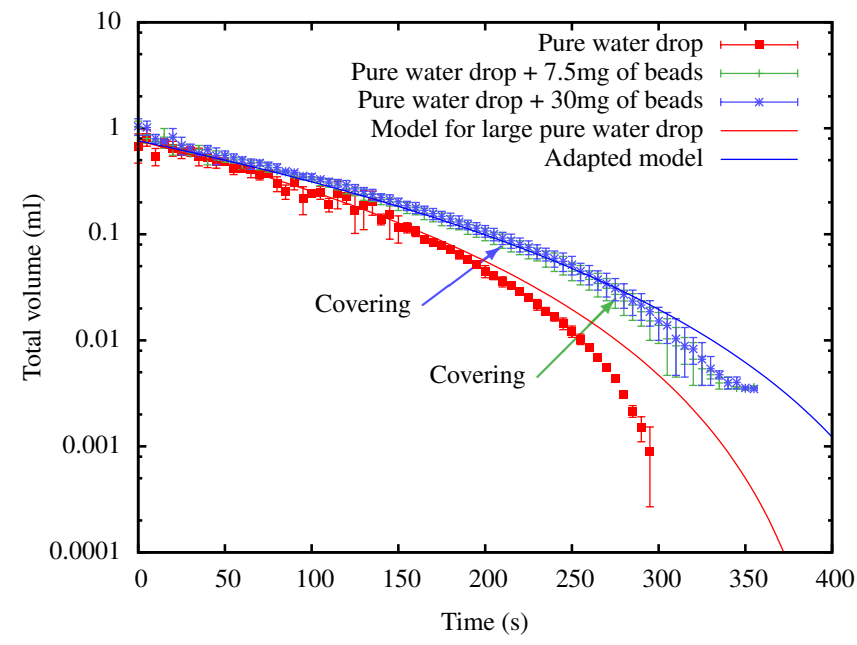

Fig. 3 Evolution of the volume as a function of time for pure water drops (red squares) and drops loaded with $7.5 \pm 0.3 \mathrm{mg}$ of grains (green crosses) and $30 \pm 0.3 \mathrm{mg}$ of grains (blue stars). The grains used are glass beads from sample II. The plate temperature was $300^{\circ} \mathrm{C}$. The red line is a fit of the evolution of the radius of pure water drops with Eq.(7) $\left(\rho_{b}=\rho_{l}, R>l_{c}\right.$, i.e. $\left.t<200 \mathrm{~s}\right)$. The blue line corresponds to the adapted model Eq.(7) $\left(\rho_{b} \neq \rho_{l}\right)$ for drops loaded with particles taking into account the reduction of the surface of evaporation.

For the case of pure liquid drops, we know that the evolution of the volume with time is separated in two regimes (radius above or below the capillary length $l_{c}=\sqrt{\gamma / g \rho_{l}}$, where $\gamma$ is the surface tension of the liquid, $\rho_{l}$ is the density of the liquid, and $g$ is the acceleration of gravity) ${ }^{18}$. We adapted the model of Biance et al. for the case of drops for which the density changes with time due to the the presence of the beads. When the radius is above the capillary length, the thickness of the vapor film $e$ can be deduced from a balance between the evaporation rate of the drop and the rate of the Poiseuille flow in the film. This balance is based on the assumption that the evaporation occurs at the bottom of the drop ${ }^{18}$. However, we first have to check that the shape of the drop, and so its surface of evaporation, is not modified by the beads for the case of our drops. To check this hypothesis, the surface of the drops as a function of their volume were compared in the case of pure water drops and water drops with beads. The results are reported in Fig. 4. Red squares and blue dots represent a drop made of pure water and a drop that carried $20 \mathrm{mg}$ of basalt grains, respectively. The beads are observed to have no effect on the shape of the drop until the moment when the drop gets fully covered. In the following, we only present the case of large drops because the drops are usually coated at radii above the capillary length, and as soon as they are coated, some buckling effects can appear and the assumption of the revolution symmetry needed to measure the volume may not be valid any longer.

Thus, we checked that the surface of contact remains the same with and without the presence of particles. As expressed in Ref. ${ }^{18}$, the balance between the mass lost by the drop due to the evaporation imposed by the heat transfer in the vapor film and the mass drained in the vapor film due to Poiseuille flow is given by

$$
\frac{\lambda_{v}}{L} \frac{\Delta T}{e} \pi R^{2} \simeq \rho_{v} \frac{2 \pi e^{3}}{3 \eta_{v}} \Delta P
$$

where $\Delta T$ is the difference of temperature between the substrate and the drop, which is assumed to be at the boiling temperature of the liquid, $L$ is the latent heat of evaporation of the liquid, and $\lambda_{v}, \eta_{v}$ and $\rho_{v}$ are the thermal conductivity, the dynamic viscosity and the density of the vapor (resp.). In the case of large drops (i.e. $R>l_{c}$ ), the pressure $\Delta P$ imposed by the drop is the hydrostatic pressure $\rho_{d} g h$, where $h$ is the height of the drop ( $\sim 2 l_{c}$ for large drops) and $\rho_{d}$ is the global density of the drop, given by

$$
\rho_{d}=\rho_{l}\left(1+\frac{V_{b}}{V_{d}} \frac{\rho_{b}-\rho_{l}}{\rho_{l}}\right)
$$

where $V_{d}$ and $V_{b}$ are the volumes of the whole drop and of the beads (resp.). Thus, the thickness of the film is given by

$$
e \simeq\left(\frac{3 \lambda_{v} \eta_{v} \Delta T}{4 \rho_{v} \rho_{d} g l_{c} L}\right)^{1 / 4} R^{1 / 2}
$$

Solving the equation of the evaporation of the drop leads to a differential equation that has no analytical solution for $\rho_{b} \neq \rho_{l}$. 


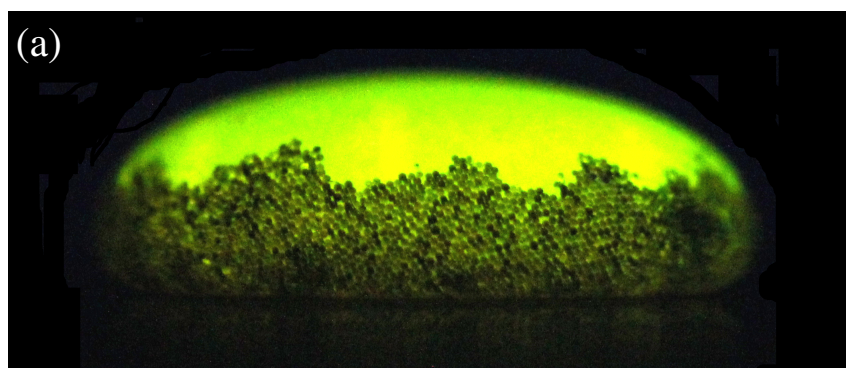

(b)

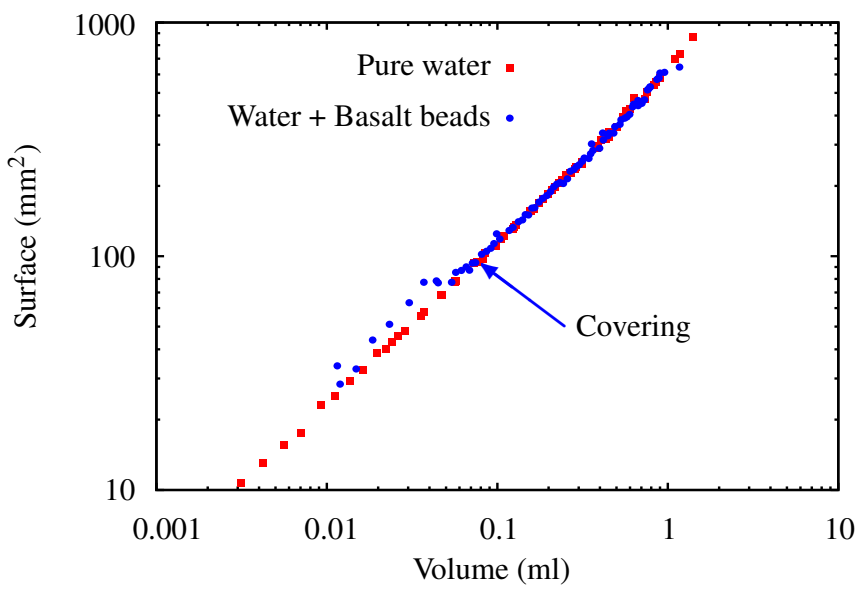

Fig. 4 (a) Image of a Leidenfrost drop in a side view. This kind of images with fluorescein enables the extraction of the surface fraction characterizing the packing of the beads $\phi$. Similar images with a backlight enable the extraction of the profile using image analysis and both the volume $V_{d}$ and the surface $S_{d r o p}$ can be deduced. (b) Comparison of the surface of Leidenfrost drops as a function of their volume in the case of a pure water drop (in red) and a drop loaded with $20 \mathrm{mg}$ of basalt beads (sample IV, in blue).

$$
\frac{\mathrm{d} V_{d}}{\mathrm{~d} t} \simeq-B V_{d}^{3 / 4}\left(1+\frac{V_{b}}{V_{d}} \frac{\rho_{b}-\rho_{l}}{\rho_{l}}\right)^{1 / 4}
$$

where

$$
B=\left(\frac{\lambda_{v} \Delta T}{L l_{c} \rho_{l}}\right)^{3 / 4}\left(\frac{\pi g \rho_{v} l_{c}}{6 \eta_{v}}\right)^{1 / 4}
$$

However, when we solve this equation in the present case, the influence of the beads on the evaporation rate is small for large drops despite the change of density, and it even increases the evaporation rate. Indeed, if the drop gets more dense, $\Delta P$ increases, leading to a decrease of the vapor film thickness, and an increase of the evaporation rate. To explain the decrease of the evaporation rate showed in Fig. 3, we need to take into account the reduction of the surface of evaporation by the partially dewetted beads. To do this, we need to replace $\pi R^{2}$ in Eq. 4 by $\pi R^{2} \phi_{\text {free }}$ where $\phi_{\text {free }}$ is the ratio between the surface free for evaporation and the whole bottom surface of the drop. This leads to

$$
B=\left(\frac{\lambda_{v} \Delta T}{L l_{c} \rho_{l}}\right)^{3 / 4}\left(\frac{\pi g \rho_{v} l_{c}}{6 \eta_{v}}\right)^{1 / 4} \phi_{\text {free }}^{3 / 4}
$$

Experimentally, when the substrate is at $300^{\circ} \mathrm{C}$, we observed that the evaporation rate is divided by about 1.4 when the drop is loaded with glass beads (sample II). This is shown by the blue line in Fig. 3. The red line is a fit on the parameter $B$ of the data for large pure water drop by the model of Biance et al. ${ }^{18}$, i.e. Eq. 9 with $\rho_{b}=\rho_{l}$, leading to $V_{d} \sim\left(t^{*}-t\right)^{4}$. Furthermore, we can see that the quantity of beads does not seem to affect the evaporation rate. This is likely because in both cases presented, the beads cover quickly the bottom surface of these drops, and so, reduce the free surface of evaporation in the same way.

This result contrasts with the case of liquid marbles for which the evaporation is faster than in the case of bare drops ${ }^{26}$. Although this fact could deserve more attention, we think this is due to the fact that the evaporation of liquid marbles at room temperature by a gradient of vapor concentration and is not much sensitive to the presence of beads ${ }^{26}$. The evaporation of Leidenfrost drops is directly affected by the reduction of the surface of evaporation.

From the reduction of the evaporation rate, we can calculate

$$
\phi_{\text {free }}=\left[B / B_{\text {pure }}\right]^{4 / 3}
$$

for each kind of considered grains. The results are presented in Table 2 for different samples of beads and two different temperatures. The quantity $\phi_{\text {free }}$ can also be estimated using geometrical arguments. In Section 3.1, we determined by image analysis that the grains apparently occupied the drop surface with a surface fraction $\phi_{0}$ (see Fig. 5a). On the other hand, the grains float: only a small part of the grains emerge (see Fig. 5c). In other words, the surface unoccupied by the beads is smaller than suggested by the side-view pictures of the droplets. In Section 2, we explained how we estimated the contact angle $\Theta_{d}$ of the liquid on the beads. Consequently, the area of the interface occupied by one grain is not $\pi R_{b}^{2}$ but $\pi\left(R_{b} \cos \left(\pi / 2-\Theta_{d}\right)\right)^{2}$. Consequently, the fraction area $\phi_{o c c}$ occupied by the grains at the interface has to be corrected by a factor $\cos ^{2} \theta$, namely

$$
\phi_{o c c}=\phi_{0} \cos ^{2} \theta
$$

with $\theta$ the complementary angle of $\Theta_{d}$. 
(a)

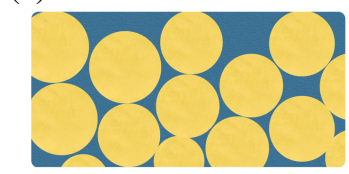

(b)

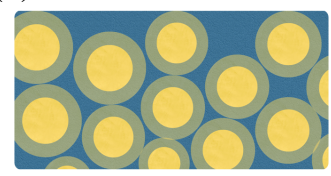

(c)

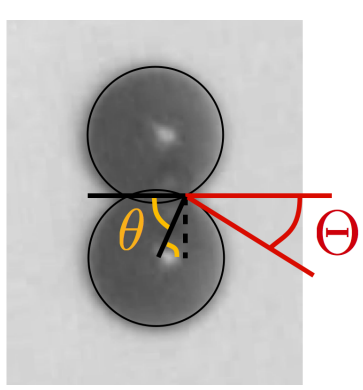

Fig. 5 The beads at the surface of a Leidenfrost drop experience dewetting and reduce the free surface of evaporation.

(a) Representation of the surface considering a contact angle of $90^{\circ}$. (b) Representation of the surface taking into account dewetting.

(c) The contact angle $\Theta$ can be calculated knowing how the surface of evaporation is modified by the beads. The picture shows a basalt bead at the interface of water in a container used to measure $\Theta_{d}$ in Table 1 and 2.

We used the values of $\phi_{\text {free }}$ obtained by Eq.(10) to estimate indirectly the angle of contact $\Theta_{i}$, namely

$$
\cos \Theta_{i}=\sqrt{\frac{\phi_{\text {free }}-1+\phi_{0}}{\phi_{0}}}
$$

The results $\phi_{\text {free }}, \Theta_{d}$ and $\Theta_{i}$ are reported in Table 2 for different samples of beads and two different temperatures. These values does not depend significantly on the temperature of the substrate, and thus, are not significantly affected by the rate of evaporation. The difference between the corresponding values of $\Theta_{d}$ and $\Theta_{i}$ can be explained by the contact angle hysteresis. Indeed, in the case of $\Theta_{i}$, the upper phase is the water. It is the opposite in the case of $\Theta_{d}$, and therefore, the contact angle can be pulled to its maximum value. Moreover, as $\Theta_{i}$ is measured in an evaporative state, while $\Theta_{d}$ is measured in an equilibrium state, one cannot exclude either an effect of apparent contact angle increase by evaporation ${ }^{28}$.

Note that the argumentation is only true when the meniscus formed at the contact line can be neglected ${ }^{27}$. The beads used in our experiments are similar in densities and in sizes than those used by Raux et al. ${ }^{27}$ and the meniscus can thus be neglected.

\section{Conclusion}

We showed that solid hydrophilic particles such as micrometric glass beads are quickly trapped by the surface of a Leidenfrost drop when they are introduced in it (e.g. from the top, as done here). The particles self-organize in the drop as the evaporation occurs. As the surface of the drop is reduced, they

\begin{tabular}{|c|c|c|c|c|}
\hline Beads & $T\left({ }^{\circ} \mathrm{C}\right)$ & $\phi_{\text {free }}$ & $\Theta_{i}$ & $\Theta_{d}$ \\
\hline \hline Pure & 300 & 1 & - & - \\
\hline Pure & 350 & 1 & - & - \\
\hline I & 300 & 0.572 & $47^{\circ}$ & - \\
\hline I & 350 & 0.612 & $44^{\circ}$ & - \\
\hline II & 300 & 0.640 & $42^{\circ}$ & $26^{\circ}$ \\
\hline II & 350 & 0.618 & $44^{\circ}$ & $26^{\circ}$ \\
\hline III & 300 & 0.624 & $43^{\circ}$ & $31^{\circ}$ \\
\hline III & 350 & 0.603 & $45^{\circ}$ & $31^{\circ}$ \\
\hline IV & 300 & 0.527 & $50^{\circ}$ & $30^{\circ}$ \\
\hline IV & 350 & 0.554 & $48^{\circ}$ & $30^{\circ}$ \\
\hline
\end{tabular}

Table 2 The table presents the fraction of the surface of evaporation that is free of beads experimentally determined from the measured reduction of the evaporation rate. These fractions enable the extraction of the contact angle of the beads in the drops, $\Theta_{i}$.

gradually cover a larger proportion of the surface and eventually completely cover the droplet. We also showed that at that moment, the grains form a monolayer around the droplet.

Hence, the process results in liquid marbles coated by hydrophilic particles. The conditions necessary to obtain such Leidenfrost marbles are: (i) the drop does not exhibit any chimney, i.e. its radius $R<R_{c}=9.6 \mathrm{~mm}$ for water drops, (ii) the volume of the inserted grains is much smaller than the initial volume of the drop, and (iii) the dried grains are able to float at the upper part of the droplet interface.

We adapted the model of Biance et al. ${ }^{18}$ to describe the evaporation of such drops by taking into account that the effective density of the drop increases as the drop evaporates. As this turned out to have only little influence, we also took into account the decrease of the free surface of evaporation by the dewetting of the particles. From measurements of the evaporation rate reduction by particles, we then could extract an apparent contact angle of the beads lying at the bottom surface of the drop, which is found to be coherent with independent equilibrium measurements.

\section{Acknowledgments}

The authors would like to thank Florian Moreau for useful advice at the early stage of the work. SD and PC are grateful to F.R.S.-FNRS for financial support. This research has been funded by the Interuniversity Attraction Pole Programme (IAP 7/38 MicroMAST) initiated by the Belgian Science Policy Office, and by the ODILE FRFC contract 2.4623 funded by F.R.S.-FNRS. 


\section{References}

1 J. G. Leidenfrost, De Aquae Communis Nonnullis Qualitatibus Tractatus, Duisburg, 1756.

2 D. Quéré, Annu. Rev. Fluid Mech., 2013, 45, 197-215.

3 H. Linke, B. J. Alemán, L. D. Melling, M. J. Taormina, M. J. Francis, C. C. Dow-Hygelund, V. Narayanan, R. P. Taylor and A. Stout, Phys. Rev. Lett., 2006, 96, 154502-4.

4 G. Dupeux, M. L. Merrer, G. Lagubeau, C. Clanet, S. Hardt and D. Quéré, Europhys. Lett., 2011, 96, 58001.

5 A. G. Marín, D. A. del Cerro, G. R. B. E. Römer, B. Pathiraj, A. H. in 't Veld and D. Lohse, Phys. Fluids, 2012, 24, 122001.

6 A. Hashmi, Y. Xu, B. Coder, P. A. Osborne, J. Spafford, G. E. Michael, G. Yu and J. Xu, Sci. Rep., 2012, $2,797$.

7 J. D. Bernardin and I. Mudawar, Trans. ASME, 1999, 121, 894-903.

8 H. Kim, B. Truong, J. Buongiorno and L.-W. Hu, Appl. Phys. Lett., 2011, 98, 083121-3.

9 T. Tran, H. J. J. Staat, A. Prosperetti, C. Sun and D. Lohse, Phys. Rev. Lett., 2012, 108, 036101-5.

10 J. H. Snoeijer, P. Brunet and J. Eggers, Phys. Rev. E, 2009, 79, 036307.

11 Y. Pomeau, M. Le Berre, F. Celestini and T. Frisch, C. R. Mec., 2012, 340, 867-881.

12 P. Brunet and J. Snoeijer, Eur. Phys. J. Special Topics, 2011, 192, 207226.

13 N. Tsapis, E. R. Dufresne, S. S. Sinha, C. S. Riera, J. W. Hutchinson, L. Mahadevan and D. A. Weitz, Phys. Rev. Lett., 2005, 94, 018302-4.

14 M. Elbahri, D. Paretkar, K. Hirmas, S. Jebril and R. Adelung, Adv. Mater., 2007, 19, 1262-1266.

15 P. Aussillous and D. Quéré, Nature, 2001, 411, 924-927.

16 G. McHale and M. I. Newton, Soft Matter, 2011, 7, 5473-5481.

17 C. Aberle, M. Lewis, G. Yu, N. Lei and J. Xu, P. ASME, 2011, 10, 643647.

18 A.-L. Biance, C. Clanet and D. Quéré, Phys. Fluids, 2003, 15, 16321637.

19 A. L. Sumner, E. J. Menke, Y. Dubowski, J. T. Newberg, R. M. Penner, J. C. Hemminger, L. M. Wingen, T. Brauers and B. J. Finlayson-Pitts, Phys. Chem. Chem. Phys., 2004, 6, 604-613.

20 K. L. Mittal, Advances in contact angle, wettability and adhesion, Scrivener, 2013, vol. 1.

21 C. P. Whitby, X. Bian and R. Sedev, Colloids Surf. A, 2013, 436, 639-646.

22 C. P. Whitby, X. Bian and R. Sedev, Soft Matter, 2012, 8, 11336-11342.

23 J. G. Berryman, Phys. Rev. A, 1983, 27, 1053-1061.

24 D. Bideau and J. P. Troadec, J. Phys. C, 1984, 17, L731.

25 X. Xu and T. Qian, Phys. Rev. E, 2013, 87, 043013-14.

26 B. Laborie, F. Lachaussee, E. Lorenceau and F. Rouyer, Soft Matter, 2013, 9, 4822-4830.

27 P. S. Raux, H. Cockenpot, M. Ramaioli, D. Quéré and C. Clanet, Langmuir, 2013, 29, 3636-3644.

28 A. Y. Rednikov, S. Rossomme and P. Colinet, Multiphase Sci. Technol., 2009, 21, 213-248.

This journal is @ The Royal Society of Chemistry [year]

Journal Name, 2010, [vol], 1-7 | 7 